\title{
False Negative probabilities in Tardos codes
}

\author{
Antonino Simone • Boris Škorić
}

\begin{abstract}
Forensic watermarking is the application of digital watermarks for the purpose of tracing unauthorized redistribution of content. The most powerful type of attack on watermarks is the collusion attack, in which multiple users compare their differently watermarked versions of the same content. Collusionresistant codes have been developed against these attacks. One of the most famous such codes is the Tardos code. It has the asymptotically optimal property that it can resist $c$ attackers with a code of length proportional to $c^{2}$.

Determining error rates for the Tardos code and its various extensions and generalizations turns out to be a nontrivial problem. In recent work we developed an approach called the Convolution and Series Expansion (CSE) method to accurately compute false positive accusation probabilities. In this paper we extend the CSE method in order to make it possible to compute false negative accusation probabilities as well.
\end{abstract}

\section{Introduction}

\subsection{Collusion attacks against forensic watermarking}

Fingerprinting provides a means for tracing the origin and distribution of digital data. Before distribution of digital content, the content is modified by applying an imperceptible fingerprint, which plays the role of a personalized serial number. The fingerprint is usually embedded through a watermarking algorithm. Once an unauthorized copy of the content is found, the identity can be determined of those users who participated in the creation of the unauthorized copy. This can be done using a tracing algorithm, which outputs a list of allegedly guilty users. This process is also known as 'forensic watermarking'.

Reliable tracing of content requires security against attacks that aim to remove the embedded information from a copy. Collusion attacks, where a group of pirates collude to compare their copies, are a particular threat. As any differences between the copies have to arise from the watermarks and not the content, such a comparison gives information which can be used to remove the watermark. To counter this threat, coding theory has produced a number of collusion-resistant codes. In any practical implementation, they must be combined with some kind of embedding scheme. The resulting system has two layers [10,21]: The coding layer determines which message to embed and protects against collusion attacks. The underlying watermarking layer hides symbols of the message in segments of the content. The symbols are either binary or from a larger alphabet. The interface between the fingerprinting code and the watermarking system is usually specified in terms of the marking assumption plus additional assumptions that are referred to as a 'model'. The marking assumption states that the colluders are able to perform modifications only in those content segments where the colluders received differently marked content. These segments are called detectable positions. The 'model' specifies the kind of symbol manipulations that the attackers are able to perform in detectable positions. The commonly used Restricted Digit Model (RDM) only allows them to 
choose pieces from their copies of the content, i.e. each segment of the unauthorized copy carries exactly one symbol that the attackers have available.

\subsection{Tardos codes}

Many collusion resistant codes have been proposed in the literature. Most notable are the Boneh-Shaw construction [4] and the by now famous Tardos code [26]. The former construction uses a concatenation of an inner code with a random outer code, while the latter one is a fully randomized binary code. G. Tardos' original work was followed by a large number of papers giving generalizations, construction improvements, sharper analysis etc. (e.g. [2,6-8,13-15,18,19,27-30]). We briefly summarize some of the developments. The number of users is $n$. The coalition size that should be resisted by the code is denoted as $c_{0}$, and $\varepsilon_{1}$ is the maximum allowed probability of accusing a fixed innocent user. In G. Tardos' original paper [26] a binary code was given achieving length $m=100 c_{0}^{2}\left\lceil\ln \frac{1}{\varepsilon_{1}}\right\rceil$, along with a proof that $m \propto c_{0}^{2}$ is asympotically optimal $^{1}$ for large coalitions, for all alphabet sizes. (The $\varepsilon_{1}$ is a function of $n$ and the maximum tolerable overall false accusation probability.) The original Tardos code construction contained two unfortunate design choices which caused the high proportionality constant ' 100 '. First, the false negative probability $\varepsilon_{2}$ (not accusing any of the guilty users) was coupled to $\varepsilon_{1}$ according to $\varepsilon_{2}=\varepsilon_{1}^{c_{0} / 4}$. This gives $\varepsilon_{2} \ll \varepsilon_{1}$ which is highly unusual in the context of content distribution; a deterring effect is achieved already at $\varepsilon_{2} \approx \frac{1}{2}$, while the false positive probability $\left(\approx n \varepsilon_{1}\right)$ needs to be very small. In the subsequent literature (e.g. [2,29]) the $\varepsilon_{2}$ was decoupled from $\varepsilon_{1}$, leading to a substantial improvement of the code length. Second, the symbols 0 and 1 were not treated on an equal footing. Only segments where the attackers produce a 1 were taken into account. This procedure ignores $50 \%$ of all the available information. A fully symbol-symmetric version of the Tardos code was given in [27], leading to a further improvement of the code length by a factor 4 .

A further improvement was achieved in [19]. The Tardos code construction consists of two probabilistic steps. In the first step, a bias parameter is generated for each segment. In Tardos' original construction the probability density function (pdf) for the bias is a continuous function, suitable for arbitrary coalition size. In [19] a class of discrete distributions was given that performs better against finite coalition sizes than the original pdf.

All the above mentioned work followed the so-called 'simple decoder' approach, i.e. an accusation score is computed for each user independently, and if it exceeds a certain threshold, the user is considered suspicious. In contrast, one can also use a 'joint decoder' which considers sets of users $[5,16]$.

Amiri and Tardos [1] have given a capacity-achieving joint decoder construction for the binary code. (Capacity refers to the information-theoretic treatment $[3,11,17,25]$ of the colluder attack as a communication channel.) However, the construction is rather impractical, requiring computations for many candidate coalitions. Even if more practical joint decoders are found, the simple decoder will serve as a stepping stone in their operation. Thus, there is considerable interest in the simple decoder.

In [27] the binary construction was generalized to alphabets of arbitrary size $q$, in the simple decoder approach. It was shown that, in the restricted digit model, the transition to a larger alphabet size has benefits beyond the mere fact that a $q$-ary symbol carries $\log _{2} q$ bits of information.

\subsection{Exact computation of the error rates of Tardos codes}

The so-called 'Gaussian approximation' or 'Gaussian assumption', introduced in [29], has been a useful tool in the analysis of Tardos codes. The assumption is that the pdf of a user's accusation score has a normal distribution. When this is the case, the statistical analysis of the code's performance can be drastically simplified; the performance is almost completely determined by a single parameter, namely the average score $\tilde{\mu}$ of the coalition.

\footnotetext{
1 The proportionality $m \propto c_{0}^{2}$ was already known in the context of spread-spectrum watermarking. Kilian et al. [12] showed that, if the watermarks have a component-wise normal distribution, then $\Omega(\sqrt{m / \ln n})$ differently marked copies are required to successfully erase any mark with non-negligible probability.
} 
The Gaussian assumption is motivated by the Central Limit Theorem (CLT): A user accusation consists of a sum of per-segment contributions, which are independent and identically distributed (i.i.d.). When many of these get added together, the result is close to normal-distributed, i.e. the pdf is very close to a Gaussian in a certain region around the average, and deviates in the tails. The longer the code becomes (i.e. the larger the coalition size $c_{0}$ ), the wider this central region. In [29] and [27] theoretical results were provided arguing that the central region is sufficiently wide to allow for application of the Gaussian approximation for realistic parameter choices. However, these arguments are not very precise.

In [22-24] an in-depth analytical and numerical investigation of the Gaussian approximation was given in the RDM case. The approach is based on the convolution rule for characteristic functions, and on a way to express the false accusation probability as a power series expansion in the small parameter $1 / \sqrt{m}$. This was dubbed 'the CSE method' (Convolution and Series Expansion). The advantage of the CSE method over simulations and other methods is that it yields reliable results also when the error probability of the code is very small. For instance, if the error rate is around $10^{-10}$, then a number of simulations of order at least $10^{10}$ is required to measure this rate; in contrast, the computational effort in the CSE method does not depend on the error rate. The work of $[22,23]$ showed, for various parameter settings and attack strategies, how the false positive probability has a transition from Gaussian behavior in the central region to worse-than-Gaussian power-law behavior outside the center. In [24] an overview was given of FP error rates for the main known attack strategies in the RDM, for a large part of the parameter space.

Until now the CSE method has not been applied to accusation probabilities of guilty users.

\subsection{Contributions and outline}

In this paper we adapt the CSE method so that it can be used to compute accusation probabilities of guilty users in the $q$-ary Tardos fingerprinting scheme. We present a number of consistency checks which demonstrate that the method (and our implementation) works, and we give ROC curves combining data on guilty and innocent user accusation probabilities.

The outline is as follows. In Section 2 we introduce notation, briefly summarize the $q$-ary Tardos scheme, and give a number of lemmas necessary for computing expectation values. In Section 3 we derive the probability density function for the guilty user score function in a single content segment. We study the tails and the first two moments of the distribution, and then compute the Fourier transform. Section 4 first details the adaptations necessary to make the CSE method work for guilty user scores. Then numerical results are presented.

\section{Preliminaries}

\subsection{General notation}

Vectors are denoted in boldface. Sets will be (mostly) written in calligraphic font. For a scalar $x$ and a vector $\boldsymbol{p}$, the notation $\boldsymbol{p}^{x}$ stands for $\prod_{\alpha} p_{\alpha}^{x}$. For vectors $\boldsymbol{p}, \boldsymbol{x}$, the notation $\boldsymbol{p}^{\boldsymbol{x}}$ means $\prod_{\alpha} p_{\alpha}^{x_{\alpha}}$.

Definition 1 (Generalized Beta function) Let $\boldsymbol{v}$ be a $n$-ary vector. The Beta function is defined as

$$
B(\boldsymbol{v}):=\frac{\prod_{a=1}^{n} \Gamma\left(v_{a}\right)}{\Gamma\left(\sum_{b=1}^{n} v_{b}\right)}
$$

For parameters $v_{1}, \cdots, v_{n}>0$ the Beta function has the following Dirichlet integral representation:

$$
B(\boldsymbol{v})=\int_{0}^{1} \mathrm{~d}^{n} \boldsymbol{x} \delta\left(1-\sum_{a=1}^{n} x_{a}\right) \prod_{b=1}^{n} x_{b}^{-1+v_{b}}
$$




\subsection{The $q$-ary Tardos scheme}

We briefly summarize the most important aspects of the Tardos scheme. The number of symbols in a codeword is $m$. The number of users is $n$. The alphabet is $\mathcal{Q}$, with size $q . X_{j i} \in \mathcal{Q}$ stands for the $i$ 'th symbol in the codeword of user $j$. The whole matrix of codewords is denoted as $X$.

Two-step code generation.

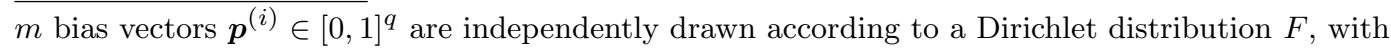

$$
F(\boldsymbol{p})=\delta\left(1-\sum_{\beta \in \mathcal{Q}} p_{\beta}\right) \cdot \frac{1}{B\left(\kappa \mathbf{1}_{q}\right)} \prod_{\alpha \in \mathcal{Q}} p_{\alpha}^{-1+\kappa}
$$

Here $\mathbf{1}_{q}$ stands for the vector $(1, \cdots, 1)$ of length $q$. All elements $X_{j i}$ are drawn independently according to $\operatorname{Pr}\left[X_{j i}=\alpha \mid \boldsymbol{p}^{(i)}\right]=p_{\alpha}^{(i)}$.

Attack.

The coalition is a subset of the set of all users. We denote the coalition as $\mathcal{C}$, with size $c$. The $i$ 'th segment of the attacked content contains a symbol $y_{i} \in \mathcal{Q}$. We define vectors $\boldsymbol{\sigma}^{(i)} \in\{0, \ldots, c\}^{q}$ as

$$
\sigma_{\alpha}^{(i)}=\left|\left\{j \in \mathcal{C}: X_{j i}=\alpha\right\}\right|
$$

satisfying $\sum_{\alpha \in \mathcal{Q}} \sigma_{\alpha}^{(i)}=c$. In words: $\sigma_{\alpha}^{(i)}$ counts how many colluders have received symbol $\alpha$ in segment $i$. For fixed $q$ and $c$, we define the set of possible $\boldsymbol{\sigma}$ values as $\mathcal{S}_{q c}=\left\{\boldsymbol{\sigma} \in\{0, \ldots, c\}^{q} \mid \sum_{\alpha \in \mathcal{Q}} \sigma_{\alpha}=c\right\}$.

The attack strategy may be nondeterministic. As usual, it is assumed that this strategy is segmentsymmetric (the same in all segments), symbol-symmetric (invariant under permutation of the alphabet) and attacker-symmetric (invariant under permutation of the attackers). The strategy is expressed as probabilities $\theta_{y \mid \boldsymbol{\sigma}}$ that apply independently for each segment. Omitting the column index,

$$
\operatorname{Pr}[y \mid \boldsymbol{\sigma}]=\theta_{y \mid \boldsymbol{\sigma}}
$$

Some often studied strategies are listed below.

\begin{tabular}{|c|c|c|c|}
\hline Strategy & Abbrev. & Description & $\theta_{y \mid \boldsymbol{\sigma}}$ \\
\hline \hline Minority Voting & MinV & Select symbol that occurs least often & \\
\hline Majority Voting & MajV & Select symbol that occurs most often & \\
\hline Interleaving & Int & Select random attacker's symbol & $\sigma_{y} / c$ \\
\hline$\tilde{\mu}$-minimizing & $\tilde{\mu}$-min & Select $\sigma_{y}>0$ that minimizes $\tilde{\mu}$ (see below) & \\
\hline Random Symbol & $\mathrm{RS}$ & Choose uniformly from received symbols & $\frac{\left[\sigma_{y}>0\right]}{\left[\alpha \in \mathcal{Q}: \sigma_{\alpha}>0\right\} \mid}$ \\
\hline
\end{tabular}

\section{Accusation.}

The watermark detector sees the symbols $y_{i}$. For each user $j$, a score $S_{j}$ is computed,

$$
S_{j}=\sum_{i=1}^{m} S_{j}^{(i)} \quad \text { where } \quad S_{j}^{(i)}=g_{\left[X_{j i}==y_{i}\right]}\left(p_{y_{i}}^{(i)}\right),
$$

where the expression $\left[X_{j i}==y_{i}\right]$ evaluates to 1 if $X_{j i}=y_{i}$ and to 0 otherwise, and the functions $g_{0}$ and $g_{1}$ are defined as

$$
g_{1}(p)=\sqrt{(1-p) / p} \quad ; \quad g_{0}(p)=-\sqrt{p /(1-p)} .
$$

The total score of the coalition is $S_{\mathcal{C}}=\sum_{j \in \mathcal{C}} S_{j}$. The choice (7) is the unique choice that, for innocent users, yields zero average accusation and variance equal to 1 independent of $\boldsymbol{p}$,

$$
p g_{1}(p)+(1-p) g_{0}(p)=0 \quad ; \quad p\left[g_{1}(p)\right]^{2}+(1-p)\left[g_{0}(p)\right]^{2}=1 .
$$

This has been shown to have optimal properties for $q=2[6,29]$. Its unique properties (8) also hold for $q \geq 3$; that is the main motivation for using (7). A user $j$ is 'accused' if his score $S_{j}$ exceeds a threshold $Z$, i.e. if $S_{j}>Z$. The list of accused users is denoted as $\mathcal{L}$. The False Positive and False negative error probability are defined as $P_{\mathrm{FP}}=\operatorname{Pr}[\mathcal{L} \backslash \mathcal{C} \neq \emptyset]$ and $P_{\mathrm{FN}}=\operatorname{Pr}[\mathcal{L} \cap \mathcal{C}=\emptyset]$.

The parameter $\tilde{\mu}$ is defined as $\frac{1}{m} \mathbb{E}[S]$, where $\mathbb{E}$ stands for the expectation value over all random variables. The $\tilde{\mu}$ depends on $q, \kappa$, the collusion strategy, and weakly on $c$. In the limit of large $c$ it converges to a finite value, and the code length scales as $m \propto c^{2} / \tilde{\mu}^{2}$. 


\subsection{Expectation values}

We will need to compute expectation values over several of the random variables mentioned above. To this end we list a number of lemmas, most of which are from the literature.

Expectation over $\boldsymbol{p}$ : Let $r(\boldsymbol{p})$ be an arbitrary function. Then the expectation over $\boldsymbol{p}$ is defined as

$$
\mathbb{E}_{\boldsymbol{p}}[r(\boldsymbol{p})]:=\int_{0}^{1} \mathrm{~d}^{q} \boldsymbol{p} F(\boldsymbol{p}) r(\boldsymbol{p}) .
$$

The following lemma is helpful when one component of $\boldsymbol{p}$ has a special status, for instance $p_{y}$, with $y$ the symbol chosen by the attackers. The rest of $\boldsymbol{p}$ is denoted as $\boldsymbol{p}_{\backslash y}$.

Lemma 1 (Marginals of the Dirichlet distribution) Let $r$ be any function of $\boldsymbol{p}$. The expectation value $\mathbb{E}_{\boldsymbol{p}}$ can be split into two parts as

$$
\mathbb{E}_{\boldsymbol{p}}[r(\boldsymbol{p})]=\mathbb{E}_{p_{y}}\left[\mathbb{E}_{\boldsymbol{p}_{\backslash y} \mid p_{y}}[r(\boldsymbol{p})]\right]
$$

with

$$
\begin{aligned}
\mathbb{E}_{p_{y}}[\cdots] & =\frac{1}{B(\kappa, \kappa[q-1])} \int_{0}^{1} \mathrm{~d} p_{y} p_{y}^{-1+\kappa}\left(1-p_{y}\right)^{-1+\kappa[q-1]}[\cdots] \\
\mathbb{E}_{\boldsymbol{p}_{\backslash y} \mid p_{y}}[r(\boldsymbol{p})] & =\left.\frac{1}{B\left(\kappa \mathbf{1}_{q-1}\right)} \int_{0}^{1} \mathrm{~d}^{q-1} \boldsymbol{t} \delta\left(1-\sum_{\beta \in \mathcal{Q} \backslash\{y\}} t_{\beta}\right) \boldsymbol{t}^{-1+\kappa} r(\boldsymbol{p})\right|_{\boldsymbol{p}_{\backslash y}=\left(1-p_{y}\right) \boldsymbol{t}}
\end{aligned}
$$

Proof: See Appendix A

Expectation over $\boldsymbol{\sigma} \mid \boldsymbol{p}$ : Let $r(\boldsymbol{\sigma})$ be an arbitrary function. Then

$$
\mathbb{E}_{\boldsymbol{\sigma} \mid \boldsymbol{p}}[r(\boldsymbol{\sigma})]:=\sum_{\boldsymbol{\sigma} \in \mathcal{S}_{q c}}\left(\begin{array}{c}
c \\
\boldsymbol{\sigma}
\end{array}\right) \boldsymbol{p}^{\boldsymbol{\sigma}} r(\boldsymbol{\sigma})
$$

Expectation over $y \mid \boldsymbol{\sigma}$ : Let $r(y)$ be an arbitrary function. Then

$$
\mathbb{E}_{y \mid \boldsymbol{\sigma}}[r(y)]:=\sum_{y \in \mathcal{Q}} \theta_{y \mid \boldsymbol{\sigma}} r(y) .
$$

Expectation over $y \mid \boldsymbol{p}$ : We introduce the notation $T_{y \mid \boldsymbol{p}}$ to denote the following sum,

$$
T_{y \mid p}=\sum_{\boldsymbol{\sigma} \in \mathcal{S}_{q c}}\left(\begin{array}{c}
c \\
\boldsymbol{\sigma}
\end{array}\right) \boldsymbol{p}^{\boldsymbol{\sigma}} \theta_{y \mid \boldsymbol{\sigma}}
$$

where the condition $\sum_{\alpha} p_{\alpha}=1$ is not enforced. This will allow us to write several important expressions compactly in terms of partial derivatives of $T$. The notation $\tau_{y \mid \boldsymbol{p}}$ is defined as $T_{y \mid \boldsymbol{p}}$ where we $d o$ enforce the 'on-shell' condition $\sum_{\alpha} p_{\alpha}=1$. It represents the conditional probability that $y$ occurs given $\boldsymbol{p}$.

$$
\mathbb{E}_{y \mid \boldsymbol{p}}[r(y)]=\sum_{y \in \mathcal{Q}} \tau_{y \mid \boldsymbol{p}} r(y)
$$

Lemma 2 (See Lemma 6 in [23]) For $d>0, v>0$, the following holds

$$
\int_{0}^{\infty} \mathrm{d} u \frac{u^{2 d-1}}{\left(1+u^{2}\right)^{d+v}}=\frac{1}{2} B(d, v) .
$$

Lemma 3 (See Lemma 3 in [23]) The overall probability distribution for one component of $\boldsymbol{\sigma}$ is

$$
\mathbb{P}_{1}(b):=\operatorname{Pr}\left[\sigma_{\alpha}=b\right]=\left(\begin{array}{l}
c \\
b
\end{array}\right) \frac{B(\kappa+b, \kappa[q-1]+c-b)}{B(\kappa, \kappa[q-1])} \quad \text { for any fixed } \alpha \in \mathcal{Q} .
$$


Corollary 1 (See Corollary 1 in [23]) Let $\boldsymbol{\sigma}_{\backslash \alpha}$ denote the vector $\boldsymbol{\sigma}$ without the component $\sigma_{\alpha}$. The probability distribution of $\boldsymbol{\sigma}_{\backslash \alpha}$ conditioned on $\sigma_{\alpha}$ is given by

$$
\mathbb{P}_{q-1}(\boldsymbol{x} \mid b):=\operatorname{Pr}\left[\boldsymbol{\sigma}_{\backslash \alpha}=\boldsymbol{x} \mid \sigma_{\alpha}=b\right]=\left(\begin{array}{c}
c-b \\
\boldsymbol{x}
\end{array}\right) \frac{B\left(\kappa \mathbf{1}_{q-1}+\boldsymbol{x}\right)}{B\left(\kappa \mathbf{1}_{q-1}\right)} \quad \text { for any fixed } \alpha \in \mathcal{Q} .
$$

Definition 2 Let $\alpha \in \mathcal{Q}, b \in\{0, \ldots, c\}, \boldsymbol{x} \in\{0, \ldots, c\}^{q-1}$ and $\boldsymbol{\sigma} \in \mathcal{S}_{q c}$ such that $\sigma_{\alpha}=b$ and $\boldsymbol{\sigma}_{\backslash \alpha}=\boldsymbol{x}$. We define

$$
\Psi_{b}(\boldsymbol{x})=\theta_{\alpha \mid \boldsymbol{\sigma}} \text { for the above given form of } \boldsymbol{\sigma} .
$$

Due to the Marking Assumption we have that $\Psi_{c}(\mathbf{0})=1$ and $\Psi_{0}(\boldsymbol{x})=0$.

Definition 3 Let $b \in\{1, \ldots, c\}$. Consider a segment for which it is given that there exists at least one symbol $\alpha \in \mathcal{Q}$ satisfying $\sigma_{\alpha}=b$, and pick one such symbol. We define $K_{b}$ as the probability that the attackers output this particular symbol.

$$
K_{b}=\mathbb{E}_{\boldsymbol{x} \mid b} \Psi_{b}(\boldsymbol{x})=\sum_{\boldsymbol{x}} \mathbb{P}_{q-1}(\boldsymbol{x} \mid b) \Psi_{b}(\boldsymbol{x}) .
$$

Lemma 4 (See Lemma 4 in [23]) The numbers $K_{b}$ satisfy

$$
q \sum_{b=1}^{c} K_{b} \mathbb{P}_{1}(b)=1 .
$$

Lemma 5 (See Theorem 2 in [23]) The expected coalition score in a single segment is

$$
\begin{aligned}
\tilde{\mu} & =\mathbb{E}\left[S_{\mathcal{C}}^{(i)}\right]=q \sum_{b=1}^{c} \mathbb{P}_{1}(b) K_{b} W(b)\left\{\frac{1}{2}-\kappa+\frac{b}{c}(\kappa q-1)\right\}, \\
\text { with } W(b) & :=c \frac{\Gamma\left(b+\kappa-\frac{1}{2}\right)}{\Gamma(b+\kappa)} \frac{\Gamma\left(c-b+\kappa[q-1]-\frac{1}{2}\right)}{\Gamma(c-b+\kappa[q-1])} .
\end{aligned}
$$

\section{Properties of the guilty-user score in a single segment}

In this section we study the properties of the single-segment score $S_{j}^{(i)}$ for a guilty user $j \in \mathcal{C}$. In Section 3.1 we derive the probability density function (pdf) $\psi$ for $S_{j}^{(i)}$, and we investigate the first two moments, as well as the tails of the distribution. In Section 3.2 we compute the Fourier transform (characteristic function) of $\psi$ and investigate its main properties, such as its power series expansion. The Fourier transform is then used in Section 4 to implement the CSE method.

\subsection{Distribution of the guilty-user score}

Throughout this section we will use the shorthand notation $u$ for $S_{j}^{(i)}$. We derive the distribution function $\psi(u)$ as follows. First we fix $\boldsymbol{p}$ and compute the conditional pdf $\psi(u \mid \boldsymbol{p})$. Then the end result follows by taking the expectation value over $\boldsymbol{p}: \psi(u)=\mathbb{E}_{\boldsymbol{p}}[\psi(u \mid \boldsymbol{p})]$. Because of the different behavior of positive and negative scores we introduce the notation $\psi_{+}$for $u>0$ and $\psi_{-}$for $u<0$.

Theorem 1 Let $T_{y \mid \boldsymbol{p}}$ and $\tau_{y \mid \boldsymbol{p}}$ be functions as defined in Section 2.3. For a guilty user, the probability distribution of the score conditioned on $\boldsymbol{p}$ is given by

$$
\begin{aligned}
u<0: \quad \psi_{-}(u \mid \boldsymbol{p}) & =\sum_{y \in \mathcal{Q}} \delta\left(u-g_{0}\left(p_{y}\right)\right) \sum_{\boldsymbol{\sigma}}\left(\begin{array}{c}
c \\
\boldsymbol{\sigma}
\end{array}\right)\left(1-\frac{\sigma_{y}}{c}\right) \boldsymbol{p}^{\boldsymbol{\sigma}} \theta_{y \mid \boldsymbol{\sigma}} \\
& =\sum_{y \in \mathcal{Q}} \delta\left(u-g_{0}\left(p_{y}\right)\right)\left[\tau_{y \mid \boldsymbol{p}}-\frac{p_{y}}{c} \frac{\partial T_{y \mid \boldsymbol{p}}}{\partial p_{y}}\right], \\
u>0: \quad \psi_{+}(u \mid \boldsymbol{p}) & =\sum_{y \in \mathcal{Q}} \delta\left(u-g_{1}\left(p_{y}\right)\right) \sum_{\boldsymbol{\sigma}}\left(\begin{array}{c}
c \\
\boldsymbol{\sigma}
\end{array}\right) \frac{\sigma_{y}}{c} \boldsymbol{p}^{\boldsymbol{\sigma}_{\theta_{y} \mid \boldsymbol{\sigma}}} \\
& =\frac{1}{c} \sum_{y \in \mathcal{Q}} \delta\left(u-g_{1}\left(p_{y}\right)\right) p_{y} \frac{\partial T_{y \mid \boldsymbol{p}}}{\partial p_{y}} .
\end{aligned}
$$


Proof: See Appendix B.

Theorem 2 For a guilty user, the distribution function $\psi$ of the score in one segment is given by

$$
\begin{array}{ll}
u<0: \quad \psi_{-}(u)=\frac{2 q}{B(\kappa, \kappa[q-1])} \sum_{b=1}^{c-1}\left(1-\frac{b}{c}\right)\left(\begin{array}{l}
c \\
b
\end{array}\right) \frac{\left(u^{2}\right)^{b+\kappa-\frac{1}{2}}}{\left(1+u^{2}\right)^{c+\kappa q}} K_{b}, \\
u>0: \quad \psi_{+}(u)=\frac{2 q}{B(\kappa, \kappa[q-1])} \sum_{b=1}^{c} \frac{b}{c}\left(\begin{array}{l}
c \\
b
\end{array}\right) \frac{\left(u^{2}\right)^{c-b+\kappa[q-1]-\frac{1}{2}}}{\left(1+u^{2}\right)^{c+\kappa q}} K_{b} .
\end{array}
$$

Proof: See Appendix C.

The expressions $(29,30)$ are rather complicated. We have double-checked their correctness by verifying the normalization and the first moment.

Consistency check 1 The function $\psi(u)$ given in Theorem 2 is correctly normalized, $\int_{-\infty}^{\infty} \mathrm{d} u \psi(u)=1$.

Proof: See Appendix D.

Consistency check 2 The function $\psi(u)$ has the correct first moment, $\int_{-\infty}^{\infty} \mathrm{d} u \psi(u) u=\tilde{\mu} / c$.

Proof: See Appendix E.

\begin{tabular}{|c|c|c|c|}
\hline Left tail & Right tail & $u \uparrow 0$ & $u \downarrow 0$ \\
\hline \hline$\frac{K_{c-1}}{|u|^{3+2 \kappa[q-1]}}$ & $\frac{K_{1}}{u^{3+2 \kappa}}$ & $K_{1}(c-1)|u|^{1+2 \kappa}$ & $u^{-1+2 \kappa[q-1]}$ \\
\hline
\end{tabular}

Table 1 Dominant powers of $\psi(u)$ in the tails and near $u=0$. All the values above are multiplied by $\frac{2 q}{B(\kappa, \kappa[q-1])}$.

The behavior in the tails and near $u=0$ is summarized in Table 1 . The right tail is dominated by the $b=1$ term; it is proportional to $(1 / u)^{3+2 \kappa}$. The integral $\int_{0}^{\infty} \mathrm{d} u \psi_{+}(u) u^{a}$ converges for $a<2+2 \kappa$. The left tail is dominated by the $b=c-1$ term, and is proportional to $(1 /|u|)^{3+2 \kappa[q-1]}$. The integral $\int_{-\infty}^{0} \mathrm{~d} u \psi_{-}(u)|u|^{a}$ converges for $a<2+2 \kappa[q-1]$. Hence, for $\kappa \in\left(0, \frac{1}{2}\right)$, the usual choice, the second moment always exists, but not the third absolute moment. We see that the right tail is heavier than the left tail, meaning that extreme positive scores are more likely than extreme negative scores. Such a property is obviously beneficial for accusing guilty users. In case the chosen strategy is MajV, the right tail is dominated by the $b=\lceil c / q\rceil$ term, which behaves as $(1 / u)^{2\lceil c / q\rceil+2 \kappa+1}$, which for $c>q$ decreases faster than $(1 / u)^{3+2 \kappa}$. For MinV the left tail is dominated by $b=\lfloor c / 2\rfloor$, which behaves as $(1 /|u|)^{2\lceil c / 2\rceil+2 \kappa[q-1]+1}$ and decreases faster than $(1 /|u|)^{3+2 \kappa[q-1]}$ for $c>2$. Since $K_{1}$ is the coefficient associated with the dominant power in the right tail, we find that $\mathrm{MinV}$ yields the most pronounced right tail. On the left side it is MajV, the strategy that most emphasizes $K_{c-1}$. Fig. 1 illustrates these trends.

Definition 4 We denote the second moment of the pdf $\psi$ as $M_{2}$,

$$
M_{2}:=\int_{-\infty}^{\infty} \mathrm{d} u \psi(u) u^{2}
$$

Definition 5 We denote the variance of the pdf $\psi$ as $V$,

$$
V:=M_{2}-\tilde{\mu}^{2} / c^{2} .
$$

Lemma 6 The second moment $M_{2}$ as defined in Def. 4 is given by

$$
M_{2}=q \sum_{b=1}^{c} K_{b} \mathbb{P}_{1}(b)\left[\left(1-\frac{b}{c}\right) \frac{b+\kappa}{c-b+\kappa[q-1]-1}+\frac{b}{c} \frac{c-b+\kappa[q-1]}{b+\kappa-1}\right] .
$$




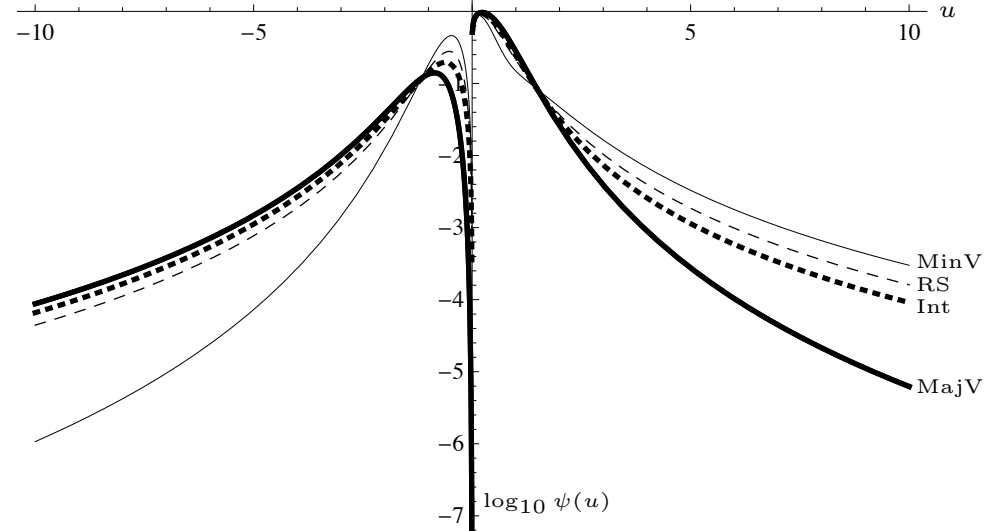

Fig. 1 The pdf $\psi$ of the single-segment score, shown for several strategies. $c=4, q=3, \kappa \approx 1 / 3$.

Proof: See Appendix F.

Remark: The scores of guilty users are not independent. As a consequence, the variance of the coalition score $S_{\mathcal{C}}^{(i)}$ is not a simple multiple of $V$. Let the covariance between two guilty user scores be $K_{j j^{\prime}}=$ $\mathbb{E}\left[S_{j}^{(i)} S_{j^{\prime}}^{(i)}\right]-\tilde{\mu}^{2} / c^{2}$. Then we have

$$
\mathbb{E}\left[\left(S_{\mathcal{C}}^{(i)}\right)^{2}\right]=\mathbb{E}\left[\sum_{j, j^{\prime} \in \mathcal{C}} S_{j}^{(i)} S_{j^{\prime}}^{(i)}\right]=c \mathbb{E}\left[\left(S_{j}^{(i)}\right)^{2}\right]+\sum_{j \neq j^{\prime}}\left(\mathbb{E}\left[S_{j}^{(i)} S_{j^{\prime}}^{(i)}\right]-\tilde{\mu}^{2} / c^{2}\right)+\tilde{\mu}^{2}\left(1-\frac{1}{c}\right) .
$$

which yields

$$
\tilde{\sigma}^{2}:=\operatorname{Var}\left(S_{\mathcal{C}}^{(i)}\right)=c V+\sum_{j \neq j^{\prime}} K_{j j^{\prime}}
$$

In [27] the variance was bounded as $\tilde{\sigma}^{2}<q c-\tilde{\mu}^{2}$. From this bound we learn that the sum $\sum_{j \neq j^{\prime}} K_{j j^{\prime}}$ scales at most linearly in $c$, even though it contains two sums over the coalition. A study of the covariances is left for future work.

Remark: In the case of the Interleaving attack, we have $\Psi_{b}(\boldsymbol{x})=b / c$ and $K_{b}=b / c$. The $\sum_{b}$ summations in Theorem 2 can be evaluated exactly, and yield

$$
\begin{aligned}
& \psi_{-}^{\text {Int }}(u)=\left(1-\frac{1}{c}\right) \frac{2 q}{B(\kappa, \kappa[q-1])} \frac{\left(u^{2}\right)^{\kappa+1 / 2}}{\left(1+u^{2}\right)^{2+\kappa q}} \\
& \psi_{+}^{\text {Int }}(u)=\frac{2 q}{B(\kappa, \kappa[q-1])}\left(c+u^{2}\right) \frac{\left(u^{2}\right)^{\kappa[q-1]-1 / 2}}{\left(1+u^{2}\right)^{2+\kappa q}} .
\end{aligned}
$$

The left tail has dominant power $(1 /|u|)^{3+2 \kappa[q-1]}$ and the right tail $(1 / u)^{3+2 \kappa}$, which corresponds to the longest possible tails as listed in Table 1. This does not come as a surprise; the Interleaving attack has the same tail behavior in the case of innocent users.

\subsection{Fourier transform}

Definition 6 Let $\chi: \mathbb{R} \rightarrow \mathbb{C}$ be a function. The Fourier transform of $\chi$ is denoted as $\tilde{\chi}$ and defined as

$$
\tilde{\chi}(k)=\int_{-\infty}^{\infty} \mathrm{d} x e^{-i k x} \chi(x) \quad \text { with } k \in \mathbb{R} .
$$

Lemma 7 Let $\chi$ be a probability distribution function, and $X$ a random variable with $X \sim \chi$. Then

$$
\left.\frac{\mathrm{d}^{n} \tilde{\chi}(k)}{\mathrm{d} k^{n}}\right|_{k=0}=(-i)^{n} \mathbb{E}\left[X^{n}\right] .
$$


Proof: $\frac{\mathrm{d}^{n} \tilde{\chi}(k)}{\mathrm{d} k^{n}}=\int \mathrm{d} x\left[\frac{\mathrm{d}^{n}}{\mathrm{~d} k^{n}} e^{-i k x}\right] \chi(x)=(-i)^{n} \int \mathrm{d} x x^{n} e^{-i k x} \chi(x)$. Setting $k=0$ gives the result.

Lemma 8 (From [20], section 2.5.9) Let $k \in \mathbb{R}$, Re $v>-\frac{1}{2}$, and $d>0$. Let the function $\Lambda$ be defined as the following convergent integral,

$$
\Lambda(d, v ; k):=\int_{0}^{\infty} \mathrm{d} u \frac{u^{2 d-1}}{\left(u^{2}+1\right)^{v+d}} e^{i k u}
$$

This integral can be expressed as

$$
\Lambda(d, v ; k)=(-i k)^{2 v} \Gamma(-2 v){ }_{1} F_{2}\left(v+d ; v+\frac{1}{2}, v+1 ; \frac{k^{2}}{4}\right)+\frac{1}{2} \sum_{j=0}^{\infty} \frac{(i k)^{j}}{j !} B\left(d+\frac{j}{2}, v-\frac{j}{2}\right)
$$

where ${ }_{1} F_{2}$ is a hypergeometric function.

Notice that in general $\Lambda(d, v ; k)$ is not an entire function of $k$ due to the appearance of the factor $k^{2 v}$ in the first term, which for general $v$ is not an entire function. The hypergeometric function ${ }_{1} F_{2}$ has the sum representation ${ }_{1} F_{2}\left(\alpha ; \beta_{1}, \beta_{2} ; z\right)=\sum_{j=0}^{\infty} \frac{(\alpha)_{j}}{j !\left(\beta_{1}\right)_{j}\left(\beta_{2}\right)_{j}} z^{j}$ where $(\alpha)_{j}=\alpha(\alpha+1) \cdots(\alpha+j-1)$ is the Pochhammer symbol. The radius of convergence is infinity.

Theorem 3 The Fourier transform of $\psi$ is given by

$$
\tilde{\psi}(k)=\frac{2 q}{B(\kappa, \kappa[q-1])} \sum_{b=1}^{c}\left(\begin{array}{l}
c \\
b
\end{array}\right) K_{b} \cdot\left[\left(1-\frac{b}{c}\right) \Lambda\left(d_{b}, v_{b} ; k\right)+\frac{b}{c} \Lambda\left(D_{b}, V_{b} ;-k\right)\right],
$$

with $\Lambda$ as defined in Lemma 8, and

$$
\begin{array}{rll}
d_{b}=b+\kappa & ; & v_{b}=c-b+\kappa[q-1] \\
D_{b}=c-b+\kappa[q-1] \quad & ; & V_{b}=b+\kappa .
\end{array}
$$

Proof: We use the expression for $\psi$ given in Theorem 2. The Fourier integral for the summands in $\psi_{+}$ is immediately of the form (41) and yields $\Lambda\left(D_{b}, V_{b} ;-k\right)$. The integral over the $\psi$ - terms is of the form $\int_{-\infty}^{0} \mathrm{~d} u f\left(u^{2}\right) e^{-i k u}$, which can be rewritten as $\int_{0}^{\infty} \mathrm{d} u f\left(u^{2}\right) e^{i k u}$; this too has the form (41) and yields $\Lambda\left(d_{b}, v_{b} ; k\right)$.

Corollary 2 For $q \geq 3$ and $\frac{1}{2(q-1)} \leq \kappa<\frac{1}{2}$, the $\tilde{\psi}$ has the following power series expansion,

$$
\begin{aligned}
\tilde{\psi}(k) & =1-i \frac{\tilde{\mu}}{c} k-\frac{1}{2} M_{2} k^{2}+A(-i k)^{2+2 \kappa}+O\left(k^{3}\right), \\
\text { where } A & :=\frac{2 q}{B(\kappa, \kappa[q-1])} K_{1} \Gamma(-2-2 \kappa) .
\end{aligned}
$$

Proof: Trivially $\mathbb{E}\left[u^{0}\right]=1$. From Consistency check 2 and Lemma 6 we know that $\mathbb{E}[u]=\frac{\tilde{\mu}}{c}$ and $\mathbb{E}\left[u^{2}\right]=$ $M_{2}$. Hence by Lemma 7 we have $\tilde{\psi}(0)=1, \tilde{\psi}^{\prime}(0)=-i \frac{\tilde{\mu}}{c}$ and $\tilde{\psi}^{\prime \prime}(0)=-M_{2}$. The expansion in $(44)$ is consistent with these values. After $k^{2}$ the powers can be non-integer. The next term in the series expansion is $k^{2+2 \kappa}$. The exponent comes from the application of Lemma 8 in Theorem 3: in the first term of (41) the $k^{2 v}$ factor can build irrational powers of $k$. The minimum value generated is for $V_{1}=1+\kappa$, with $V_{b}$ as defined in (43). Note that the $\Lambda$ term obtained from $v_{c}$ is not present because it is multiplied by $1-b / c$. The next contribution is $v_{c-1}=1+\kappa[q-1]$ which (for $q \geq 3$ ) is larger than $V_{1}$. Finally, the coefficient $A$ follows from the $\Lambda\left(D_{1}, V_{1},-k\right)$ term in (42), taking only the leading term $(=1)$ in the sum representation of the ${ }_{1} F_{2}$ function.

In order to apply the CSE method we will have to work with a zero-mean pdf. For this reason we introduce a 'centered' version of $\psi$. 
Definition 7 We define the pdf $\chi$ as a shifted version of $\psi$,

$$
\chi(r):=\psi\left(\frac{\tilde{\mu}}{c}+r\right)
$$

We will use shorthand notation $r=u-\tilde{\mu} / c$. From the definition it trivially follows that $\mathbb{E}[r]=0$ and $\mathbb{E}\left[r^{2}\right]=V$.

Lemma 9 The Fourier transform of $\chi$ is given by

$$
\tilde{\chi}(k)=e^{i k \frac{\tilde{\mu}}{c}} \tilde{\psi}(k) .
$$

Proof: $\tilde{\chi}(k)=\int_{-\infty}^{\infty} \mathrm{d} r e^{-i k r} \chi(r)=\int_{-\infty}^{\infty} \mathrm{d} u e^{-i k\left(u-\frac{\tilde{\mu}}{c}\right)} \psi(u)=e^{i k \frac{\tilde{\mu}}{c}} \tilde{\psi}(k)$.

Corollary 3 Let $\frac{1}{2[q-1]}<\kappa<\frac{1}{2}$ and let $\chi$ be as given in Definition $\%$. Then $\tilde{\chi}$ has the following power series expansion,

$$
\tilde{\chi}(k)=1-\frac{1}{2} V k^{2}+A(-i k)^{2+2 \kappa}+O\left(k^{3}\right)
$$

with $A$ as given in (45).

Proof: From (47) we can rewrite $\tilde{\chi}(k)$ as a product of the series expansions of $e^{i k \frac{\tilde{\mu}}{c}}$ and $\tilde{\psi}(k)$. Since $e^{i k \frac{\tilde{\mu}}{c}}=1+i \frac{\tilde{\mu}}{c} k-\frac{1}{2} \frac{\tilde{\mu}^{2}}{c^{2}} k^{2}+O\left(k^{3}\right)$, and the $\tilde{\psi}(k)$ expansion was given in (44), we have

$$
\begin{aligned}
e^{i k \frac{\tilde{\mu}}{c}} \tilde{\psi}(k) & =\left[1+i \frac{\tilde{\mu}}{c} k-\frac{1}{2} \frac{\tilde{\mu}^{2}}{c^{2}} k^{2}+O\left(k^{3}\right)\right]\left[1-i \frac{\tilde{\mu}}{c} k-\frac{1}{2} M_{2} k^{2}+A(-i k)^{2+2 \kappa}+O\left(k^{3}\right)\right] \\
& =1+0 k+\left(-\frac{M_{2}}{2}-\frac{\tilde{\mu}^{2}}{2 c^{2}}+\frac{\tilde{\mu}^{2}}{c^{2}}\right) k^{2}+A(-i k)^{2+2 \kappa}+O\left(k^{3}\right),
\end{aligned}
$$

and (48) follows after some simplification.

Remark: The $1-\frac{1}{2} V k^{2}$ part of (48) can be also found using Lemma 7 , since we know that $\mathbb{E}[r]=0$ and $\mathbb{E}\left[r^{2}\right]=V$.

In the expression (48) there are no powers between $k^{0}$ and $k^{2}$. This makes it possible for us to use the CSE method.

\section{Applying the CSE method to the guilty user score}

We are now finally in a position to compute accusation probabilities for guilty users. The Fourier transform $\tilde{\chi}$ serves as the basis; raising it to the power $m$ yields the Fourier-transformed pdf of the total accusation $S_{j}$. The computational steps are almost identical to the case of the innocent score distribution [23], with two minor differences: (i) The variance of the single-segment pdf is $V$ instead of 1; (ii) The pdf has non-zero average.

In Section 4.1 we show how these differences affect the theory. In Section 4.2 we discuss how the one-pirate probability $\operatorname{Pr}\left[S_{j}>Z\right]$ is used to obtain an upper bound on $P_{\mathrm{FP}}$. In Section 4.3 we present plots that demonstrate the convergence of our numerical implementation. Furthermore, we present an ROC curve combining innocent and guilty accusation probabilities. 


\subsection{Adaptations}

Below we list the (slight) modifications in the CSE method, as compared to [23], induced by the $V \neq 1$ variance and the nonzero mean. First, the tail of the Gaussian distribution changes.

Definition 8 We define the function $\Omega$ as the probability mass in the right tail of the normal distribution, $\Omega(z):=\frac{1}{\sqrt{2 \pi}} \int_{z}^{\infty} \mathrm{d} x e^{-x^{2} / 2}$.

Lemma 10 Let $V>0$ be the variance defined in (32). Then, for $x \in \mathbb{R}$ it holds that

$$
\frac{1}{2 \pi i} \int_{-\infty}^{\infty} \mathrm{d} k \frac{e^{i k x}}{k} e^{-\frac{V}{2} k^{2}}=\frac{1}{2}-\Omega(x / \sqrt{V})
$$

Proof: From Eq. 9.254.1 in [9] we have that $\frac{1}{2 \pi i} \int_{-\infty}^{\infty} \mathrm{d} k \frac{e^{i k x}}{k} e^{-k^{2} / 2}=\frac{1}{2}-\Omega(x)$. Changing the integration variable in (51) to $k^{\prime}=k \sqrt{V}$ immediately yields the result.

The modified Gaussian tail leads to modifications in all the integrals involving the tail.

Lemma 11 Let $V>0$ be the variance defined in (32). For $x \in \mathbb{R}$ and $\nu>0$ it holds that

$$
\int_{-\infty}^{\infty} \frac{\mathrm{d} k}{2 \pi}(i \operatorname{sgn} k)^{\alpha-1}|k|^{\nu-1} e^{-\frac{V}{2} k^{2}} e^{i k x}=\frac{1}{\pi V^{\frac{\nu}{2}}} \Gamma(\nu) 2^{\nu / 2} \operatorname{Im}\left[i^{-\alpha} H_{-\nu}\left(\frac{i x}{\sqrt{2 V}}\right)\right] .
$$

Here $\mathrm{H}_{-\nu}$ is a Hermite function.

Proof: Corollary 2 in [23] states that for $x \in \mathbb{R}$ and $\nu>0$ :

$$
\int_{-\infty}^{\infty} \frac{\mathrm{d} k}{2 \pi}(i \operatorname{sgn} k)^{\alpha-1}|k|^{\nu-1} e^{-k^{2} / 2} e^{i k x}=\frac{1}{\pi} \Gamma(\nu) 2^{\nu / 2} \operatorname{Im}\left[i^{-\alpha} H_{-\nu}\left(\frac{i x}{\sqrt{2}}\right)\right] .
$$

A change of integration variable to $k \sqrt{V}$ in (52) directly leads to the end result.

The nonzero expectation value $\mathbb{E}\left[S_{j}\right]=m \tilde{\mu} / c$ gives rise to a 'shifted' version of the formula for the accusation probability. We introduce a shifted accusation threshold $\Delta$,

$$
\Delta:=Z-m \tilde{\mu} / c \quad ; \quad \tilde{\Delta}:=\Delta / \sqrt{m} .
$$

The accusation probability can be expressed as a function of $\tilde{\Delta}$, as shown in the following two theorems.

Theorem 4 Let $j$ be a guilty user. Let $R_{m}$ denote the accusation probability $\operatorname{Pr}\left[S_{j}>Z\right]$. Then

$$
R_{m}(\tilde{\Delta})=\frac{1}{2}+\frac{i}{2 \pi} \int_{-\infty}^{\infty} \mathrm{d} k \frac{\exp i k \tilde{\Delta}}{k}\left[\tilde{\chi}\left(\frac{k}{\sqrt{m}}\right)\right]^{m}
$$

Proof: Exactly the same as the proof of (Theorem 3 in [23]), but with $\tilde{\Delta}$ replacing $\tilde{Z}$.

Theorem 5 Let $j$ be a guilty user and $\frac{1}{2[q-1]}<\kappa<\frac{1}{2}$. Then it is possible to write

$$
\left[\tilde{\chi}\left(\frac{k}{\sqrt{m}}\right)\right]^{m}=\exp \left(-\frac{1}{2} V k^{2}\right)\left[1+\sum_{t=0}^{\infty} \omega_{t}(m)(i \operatorname{sgn} k)^{\alpha_{t}}|k|^{\nu_{t}}\right]
$$

where $\alpha_{t}$ are real numbers; the coefficients $\omega_{t}(m)$ are real; the powers $\nu_{t}$ satisfy $\nu_{0}=2+2 \kappa$ and $\nu_{t+1}>\nu_{t}$. The $\nu_{t}$ are not necessarily integer. All the coefficients $\omega_{t}(m)$ are decreasing functions of $m$. The probability of accusing user $j$ is given by

$$
R_{m}(\tilde{\Delta})=\Omega(\tilde{\Delta} / \sqrt{V})+\frac{1}{\pi} \sum_{t=0}^{\infty} \omega_{t}(m) \Gamma\left(\nu_{t}\right)(2 / V)^{\nu_{t} / 2} \operatorname{Im}\left[i^{-\alpha_{t}} H_{-\nu_{t}}(i \tilde{\Delta} / \sqrt{2 V})\right] .
$$

Proof: See Appendix G. 
4.2 Relation between $\operatorname{Pr}\left[S_{j}>Z\right]$ and the False Negative probability

The quantity that we compute, $\operatorname{Pr}\left[S_{j}>Z\right]$, is not equal to the quantity we are most interested in, $P_{\mathrm{FN}}$. Below we explain how we obtain a bound on $P_{\mathrm{FN}}$ based on $\operatorname{Pr}\left[S_{j}>Z\right]$.

Lemma 12 Let $j \in \mathcal{C}$. Let $\mathcal{L}$ be the set of accused users, and $\mathcal{A}=\mathcal{L} \cap \mathcal{C}$ the set of attackers that end up in $\mathcal{L}$. Then the False Negative probability can be expressed as

$$
P_{\mathrm{FN}}=1-c \operatorname{Pr}[j \in \mathcal{L}]+(c-1) \operatorname{Pr}[\mathcal{A}=\mathcal{C}] .
$$

Proof: We start by writing

$$
\operatorname{Pr}[j \in \mathcal{L}]=\operatorname{Pr}[\mathcal{A}=\{j\}]+\sum_{k \in \mathcal{C} \backslash\{j\}} \operatorname{Pr}[\mathcal{A}=\{j, k\}]+\sum_{(k, \ell): k, \ell \in \mathcal{C} \backslash\{j\}} \operatorname{Pr}[\mathcal{A}=\{j, k, \ell\}]+\cdots+\operatorname{Pr}[\mathcal{A}=\mathcal{C}]
$$

where $(k, \ell)$ is a pair with $k \neq \ell$, and the dots denote summation over all tuplets in $\mathcal{C} \backslash\{j\}$ up to and including size $c-2$. Next we take the sum $\sum_{j \in \mathcal{C}}$ over the whole equation (59). This yields $c \operatorname{Pr}[j \in \mathcal{L}]=$ $\sum_{s=1}^{c-1} \operatorname{Pr}[|\mathcal{A}|=s]+c \operatorname{Pr}[\mathcal{A}=\mathcal{C}]$. Finally we use $1-P_{\mathrm{FN}}=\sum_{s=1}^{c} \operatorname{Pr}[|\mathcal{A}|=s]$.

The CSE method applied to one guilty user does not allow us to compute $\operatorname{Pr}[\mathcal{A}=\mathcal{C}]$. In order to upper bound the $P_{\mathrm{FN}}$ we will therefore use the following corollary.

Corollary 4 Let $j \in \mathcal{C}$. It holds that $P_{\mathrm{FN}}<1-\operatorname{Pr}\left[S_{j}>Z\right]$.

Proof: Use $\operatorname{Pr}[\mathcal{A}=\mathcal{C}]<\operatorname{Pr}[j \in \mathcal{A}]=\operatorname{Pr}[j \in \mathcal{L}]=\operatorname{Pr}\left[S_{j}>Z\right]$ in Lemma 12.

Remark. The bound provided in Corollary 4 is not always tight. Note that $\operatorname{Pr}[\mathcal{L}=\mathcal{C}] \ll \operatorname{Pr}[j \in \mathcal{L}]$ if $Z$ is 'significantly' larger than $m \tilde{\mu} / c$, yielding $P_{\mathrm{FN}} \approx 1-c \operatorname{Pr}\left[S_{j}>Z\right]$. This is a much smaller number than what Corollary 4 gives us. However, we have not been able to prove a tight upper bound on $P_{\mathrm{FN}}$.

\subsection{Numerical results}

We have implemented the CSE formulas of Section 4.1 in Wolfram Mathematica. In this section we present graphs to demonstrate that our implementation works and that we can generate ROC curves with it; we do not yet put the method to work to derive many "useful" results, e.g. exhaustive comparison of strategies for a large region of parameter space. That is left for future work.

Convergence

The convergence of (57) turns out to be rather quick. Often it suffices to take powers only up to $\nu_{t} \approx 10$ in order to get good accuracy. An example is shown in Fig. 2. (The parameters were chosen such that we are not in the Gaussian regime but in the right tail.)

Consistency check: power law in the tails

In Table 1 we see that the single-segment pdf has a power law $(1 / u)^{3+2 \kappa}$ in the right tail (provided that $\left.K_{1} \neq 0\right)$. Hence the integrated probability mass beyond $Z$ scales as $(1 / Z)^{2+2 \kappa}$. For large $Z$ we expect to see the $(1 / Z)^{2+2 \kappa}$ scaling also in the $R_{m}(\tilde{\Delta})$ curves. (Due to the Central Limit Theorem, the $R_{m}(\tilde{\Delta})$ goes to a Gaussian shape, but only for small $\tilde{\Delta}$; for large $\tilde{\Delta}$ the original single-segment tail is still there.) We use this as a consistency check on our CSE implementation. Fig. 3 shows a log-log plot of the right tail for various strategies. The tails in this plot indeed have the same slope as the curve for $m=1$.

$\underline{\text { ROC curves }}$

One of the most useful types of graph for decision-making problems is the Receiver Operating Characteristic (ROC). We take a slightly different graph, with $\varepsilon_{1}$ and (our upper bound on) $P_{\mathrm{FN}}$ on the axes. This way, being closer to the origin means better performance. An example is shown in Fig. 4. Each curve corresponds to tracing $Z$ from very low (lots of users get accused: high FP and low FN) to very high (almost nobody gets accused: low FP and high FN). The order of the curves is consistent with [24]. For the given choice of parameters, the plotted $Z$ lies outside the Gaussian regime; hence MinV is the strongest attack and $\tilde{\mu}$-min is a very poor attack. 


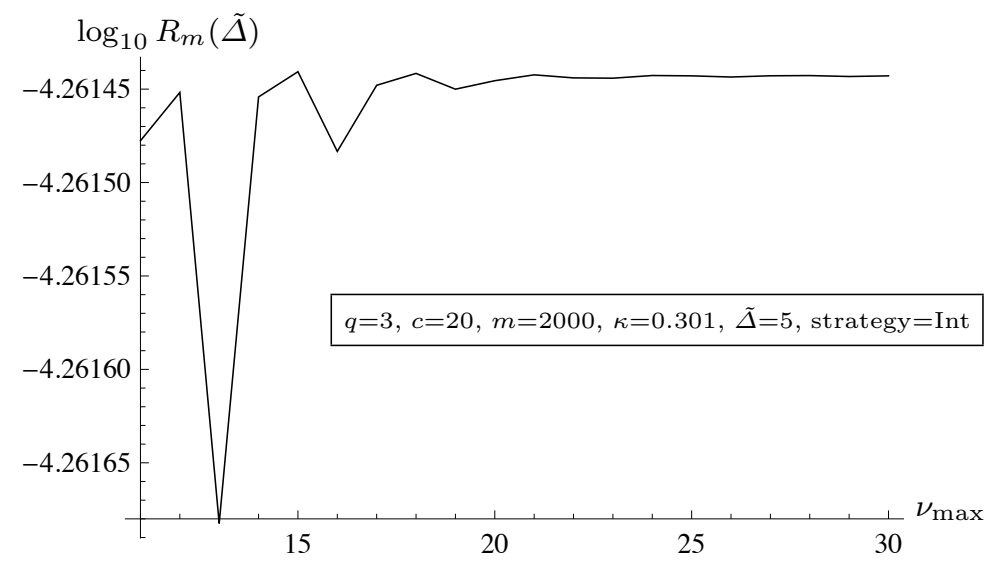

Fig. 2 Convergence example. Estimated $R_{m}(\tilde{\Delta})$ as a function of the cutoff power $\nu_{\max }$.

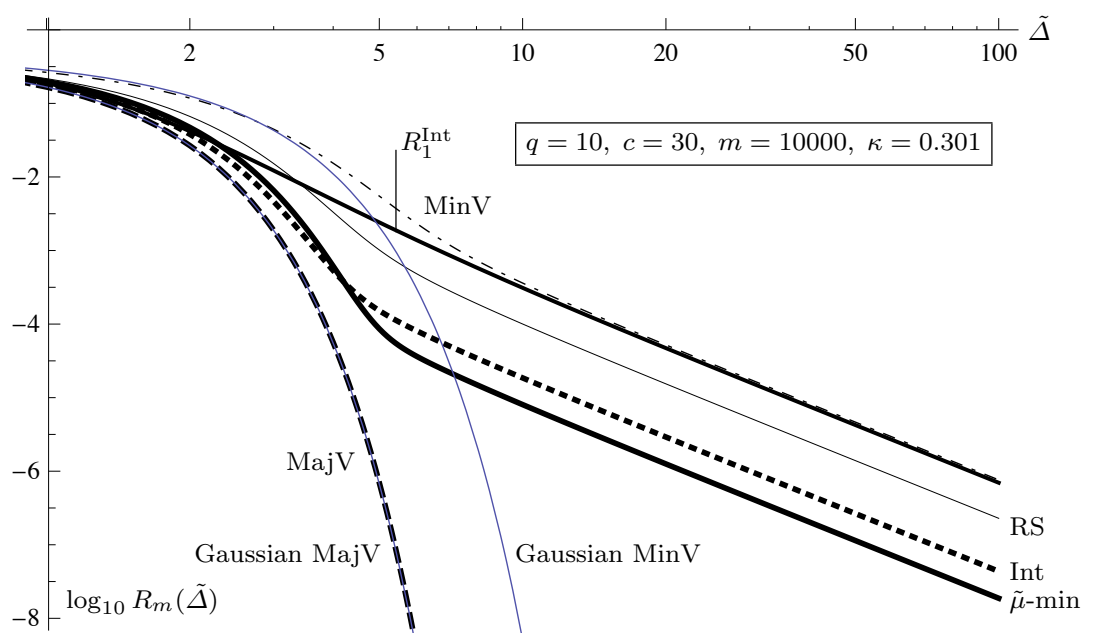

Fig. 3 Log-log plot of $R_{m}(\tilde{\Delta})$ for several strategies. The single-segment tail integral $R_{1}(\tilde{\Delta})$ for the Int attack is also shown. Two Gaussian tails are plotted: for the $V$ value corresponding to the MajV and MinV strategies. The MajV curve coincides with its Gaussian approximation.

\section{Conclusions}

We have adapted the CSE method so that we can compute guilty user accusation probabilities in the $q$-ary Tardos scheme, in the Restricted Digit Model. The main steps are the derivation of $\psi(u)$ (Theorem 2), taking the Fourier transform (Theorem 3), and executing the changes due to $\mathbb{E}[u] \neq 0$ and $\operatorname{Var}(u) \neq 1$ (Section 4.1). The rest of the work is precisely as for the innocent score pdf. We have implemented the thus adapted CSE method and done some tests. Convergence of the series expansion seems to be faster than for the innocent pdf. The large- $Z$ tails have the expected power law $(1 / \tilde{\Delta})^{2+2 \kappa}$.

Having the CSE method at our disposal for guilty as well as innocent user scores, it now becomes possible to make full ROC curves such as Fig. 4. These can serve for choosing optimal parameter settings in the Tardos scheme (even though our bound $P_{\mathrm{FN}}<\operatorname{Pr}\left[S_{\text {guilty }}<Z\right]$ is not tight). An exhaustive study of ROC curves is left for future work. 


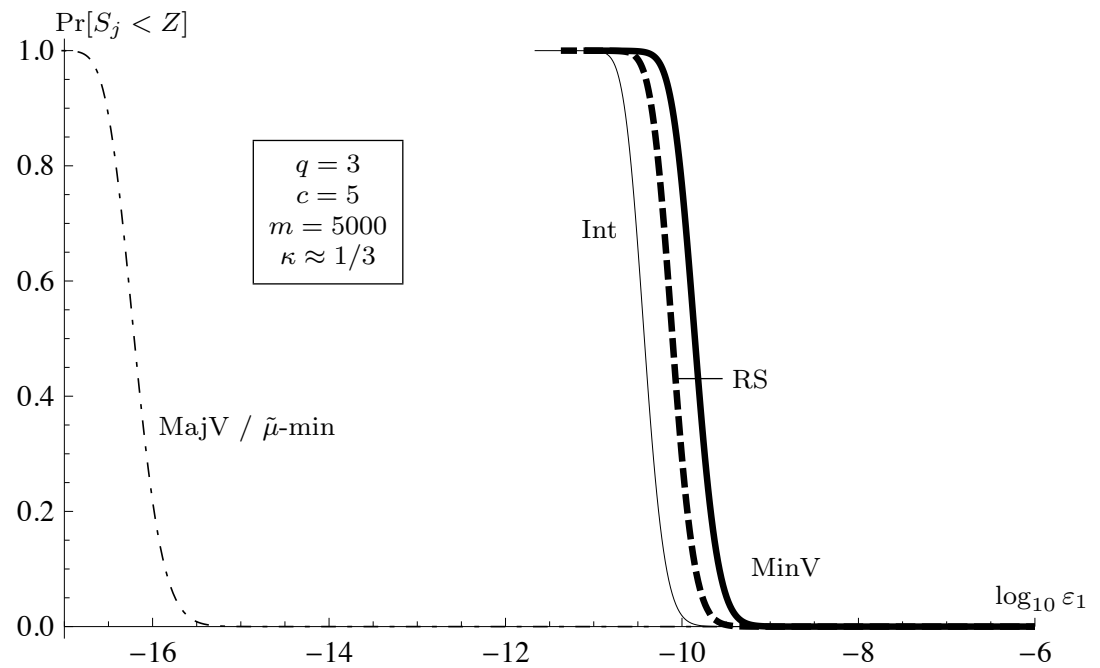

Fig. 4 Example ' $R O C$ ' curve. Our upper bound $\operatorname{Pr}\left[S_{j}<Z\right]$ on $P_{\mathrm{FN}}$ versus the probability $\varepsilon_{1}$ of accusing a fixed innocent user. The $\varepsilon_{1}$ data is taken from [24].

\section{References}

1. E. Amiri and G. Tardos. High rate fingerprinting codes and the fingerprinting capacity. In $S O D A 2009$, pages $336-345$.

2. O. Blayer and T. Tassa. Improved versions of Tardos' fingerprinting scheme. Designs, Codes and Cryptography, 48(1):79-103, 2008.

3. D. Boesten and B. Škorić. Asymptotic fingerprinting capacity for non-binary alphabets. In Information Hiding 2011, volume 6958 of Lecture Notes in Computer Science, pages 1-13. Springer, 2011.

4. D. Boneh and J. Shaw. Collusion-secure fingerprinting for digital data. IEEE Transactions on Information Theory, 44(5):1897-1905, 1998.

5. A. Charpentier, F. Xie, C. Fontaine, and T. Furon. Expectation maximization decoding of Tardos probabilistic fingerprinting code. In Media Forensics and Security, volume 7254 of SPIE Proceedings, page $72540,2009$.

6. T. Furon, A. Guyader, and F. Cérou. On the design and optimization of Tardos probabilistic fingerprinting codes. In Information Hiding, volume 5284 of LNCS, pages 341-356. Springer, 2008.

7. T. Furon and L. Pérez-Freire. Worst case attacks against binary probabilistic traitor tracing codes. In $I E E E$ Workshop on Information Forensics and Security (WIFS) 2009. IEEE. arxiv.org/abs/0903.3480.

8. T. Furon, L. Pérez-Freire, A. Guyader, and F. Cérou. Estimating the minimal length of Tardos code. In Information Hiding 2009, volume 5806 of LNCS, pages 176-190.

9. I.S. Gradshteyn and I.M. Ryzhik. Table of Integrals, Series, and Products, 5th edition. Academic Press, 1994.

10. S. He and M. Wu. Joint coding and embedding techniques for multimedia fingerprinting. TIFS, 1:231-248, 2006.

11. Y.W. Huang and P. Moulin. Saddle-point solution of the fingerprinting capacity game under the marking assumption. In ISIT 2009.

12. J. Kilian, F.T. Leighton, L.R. Matheson, T.G. Shamoon, R.E. Tarjan, and F. Zane. Resistance of digital watermarks to collusive attacks. In ISIT 1998, page 271.

13. M. Kuribayashi, N. Akashi, and M. Morii. On the systematic generation of Tardos's fingerprinting codes. In MMSP 2008, pages 748-753.

14. T. Laarhoven and B.M.M. de Weger. Optimal symmetric Tardos traitor tracing schemes, 2011. http://arxiv . org/abs/1107.3441.

15. T. Laarhoven, J. Doumen, P. Roelse, B. Škorić, and B.M.M. de Weger. Dynamic Tardos Traitor Tracing Schemes. http://arxiv.org/abs/1111.3597, 2011.

16. P. Meerwald and T. Furon. Towards Joint Tardos Decoding: The 'Don Quixote' Algorithm. In Information Hiding, pages 28-42, 2011.

17. P. Moulin. Universal fingerprinting: Capacity and random-coding exponents. In Preprint arXiv:0801.3837v2, 2008.

18. K. Nuida. Short collusion-secure fingerprint codes against three pirates. In Information Hiding, volume 6387 of $L N C S$, pages 86-102. Springer, 2010.

19. K. Nuida, M. Hagiwara, H. Watanabe, and H. Imai. Optimal probabilistic fingerprinting codes using optimal finite random variables related to numerical quadrature. $C o R R$, abs/cs/0610036, 2006.

20. A.P. Prudnikov, Yu.A. Brychkov, and O.I. Marichev. Integrals and Series, 4th printing, volume 1. CRC, 1998.

21. H.G. Schaathun. On error-correcting fingerprinting codes for use with watermarking. Multimedia Systems, $13(5-6): 331-344,2008$. 
22. A. Simone and B. Škorić. Asymptotically false-positive-maximizing attack on non-binary Tardos codes. In Information Hiding, pages 14-27, 2011.

23. A. Simone and B. Škorić. Accusation probabilities in Tardos codes: beyond the Gaussian approximation. Designs, Codes and Cryptography, 63(3):379-412, 2012.

24. A. Simone and B. Škorić. False Positive probabilities in q-ary Tardos codes: comparison of attacks. http: //eprint.iacr.org/2012/522, 2012.

25. A. Somekh-Baruch and N. Merhav. On the capacity game of private fingerprinting systems under collusion attacks. IEEE Trans. Inform. Theory, 51:884-899, 2005.

26. G. Tardos. Optimal probabilistic fingerprint codes. In STOC 2003, pages 116-125.

27. B. Škorić, S. Katzenbeisser, and M.U. Celik. Symmetric Tardos fingerprinting codes for arbitrary alphabet sizes. Designs, Codes and Cryptography, 46(2):137-166, 2008.

28. B. Škorić, S. Katzenbeisser, H.G. Schaathun, and M.U. Celik. Tardos fingerprinting codes in the combined digit model. In IEEE Workshop on Information Forensics and Security (WIFS) 2009, pages 41-45.

29. B. Škorić, T.U. Vladimirova, M.U. Celik, and J.C. Talstra. Tardos fingerprinting is better than we thought. IEEE Trans. on Inf. Theory, 54(8):3663-3676, 2008.

30. F. Xie, T. Furon, and C. Fontaine. On-off keying modulation and Tardos fingerprinting. In MMESec 2008, pages 101-106.

\section{Appendix}

\section{A Proof of Lemma 1}

The proof is similar to the steps taken in Appendix D of [23]. First we split the $q$-dimensional integration $\int \mathrm{d}^{q} \boldsymbol{p} F(\boldsymbol{p}) r(\boldsymbol{p})$ as follows,

$$
\mathbb{E}_{\boldsymbol{p}}[r(\boldsymbol{p})]=\frac{1}{B\left(\kappa \mathbf{1}_{q}\right)} \int_{0}^{1} \mathrm{~d} p_{y} p_{y}^{-1+\kappa} \int_{0}^{1-p_{y}} \mathrm{~d}^{q-1} \boldsymbol{p}_{\backslash y} \delta\left(1-p_{y}-\sum_{\beta \in \mathcal{Q} \backslash\{y\}} p_{\beta}\right) \boldsymbol{p}_{\backslash y}^{-1+\kappa} r(\boldsymbol{p}) .
$$

Then we write $\boldsymbol{p}_{\backslash y}=\left(1-p_{y}\right) \boldsymbol{t}$. We get $\delta\left(1-p_{y}-\sum_{\beta \in \mathcal{Q} \backslash\{y\}} p_{\beta}\right)=\left(1-p_{y}\right)^{-1} \delta\left(1-\sum_{\beta \in \mathcal{Q} \backslash\{y\}} t_{\beta}\right)$. Furthermore, $\mathrm{d}^{q-1} \boldsymbol{p}_{\backslash y}=\left(1-p_{y}\right)^{q-1} \mathrm{~d}^{q-1} \boldsymbol{t}$ and $\boldsymbol{p}_{\backslash y}^{-1+\kappa}=\left(1-p_{y}\right)^{(q-1)(-1+\kappa)} \boldsymbol{t}^{-1+\kappa}$. Combined with the fact that $B\left(\kappa \mathbf{1}_{q}\right)=$ $B(\kappa, \kappa[q-1]) B\left(\kappa \mathbf{1}_{q-1}\right)$, these steps yield the end result.

\section{B Proof of Theorem 1}

The guilty user's symbol is denoted as $X$. The one-segment score is either $g_{0}\left(p_{y}\right)$ (when $\left.X \neq y\right)$ or $g_{1}\left(p_{y}\right)$ (when $X=y$ ). Since no other values are possible, the probability distribution at given $\boldsymbol{p}$ will consist of delta-function peaks. Each peak is multiplied by the probability that the corresponding event occurs

$$
\begin{aligned}
& \psi_{-}(u \mid \boldsymbol{p})=\sum_{y \in \mathcal{Q}} \delta\left(u-g_{0}\left(p_{y}\right)\right) \operatorname{Pr}\left[u=g_{0}\left(p_{y}\right) \mid \boldsymbol{p}\right] \\
& \psi_{+}(u \mid \boldsymbol{p})=\sum_{y \in \mathcal{Q}} \delta\left(u-g_{1}\left(p_{y}\right)\right) \operatorname{Pr}\left[u=g_{1}\left(p_{y}\right) \mid \boldsymbol{p}\right] .
\end{aligned}
$$

Notice that

$$
\operatorname{Pr}\left[u=g_{0}\left(p_{y}\right) \mid \boldsymbol{p}\right]=\operatorname{Pr}[X \neq y \wedge Y=y \mid \boldsymbol{p}] \quad ; \quad \operatorname{Pr}\left[u=g_{1}\left(p_{y}\right) \mid \boldsymbol{p}\right]=\operatorname{Pr}[X=y \wedge Y=y \mid \boldsymbol{p}]
$$

and that

$$
\operatorname{Pr}[X \neq y \wedge Y=y \mid \boldsymbol{p}]+\operatorname{Pr}[X=y \wedge Y=y \mid \boldsymbol{p}]=\operatorname{Pr}[Y=y \mid \boldsymbol{p}]=\tau_{y \mid \boldsymbol{p}}
$$

Next step is to compute $\operatorname{Pr}\left[u=g_{1}\left(p_{y}\right) \mid \boldsymbol{p}\right]$ in (62). Let be $\boldsymbol{e}_{y}$ a $q$-ary vector entirely set to 0 except for the $y$-th element that is instead equal to 1 .

$$
\operatorname{Pr}\left[u=g_{1}\left(p_{y}\right) \mid \boldsymbol{p}\right]=\operatorname{Pr}\left[X_{j i}=y\right] \operatorname{Pr}\left[Y=y \mid X_{j i}=y, \boldsymbol{p}\right]=p_{y} \sum_{\boldsymbol{\sigma} \in \mathcal{S}_{q c}}\left(\begin{array}{c}
c-1 \\
\boldsymbol{\sigma}-\boldsymbol{e}_{y}
\end{array}\right) \boldsymbol{p}^{\boldsymbol{\sigma}-\boldsymbol{e}_{y}} \theta_{y \mid \boldsymbol{\sigma}}
$$

The last equation is obtained as follows: $\operatorname{Pr}\left[X_{j i}=y\right]=p_{y} ; \operatorname{Pr}\left[Y=y \mid X_{j i}=y, \boldsymbol{p}\right]$ is equal to the sum over all the possible $\boldsymbol{\sigma}$ vectors that have at least one occurrence of $y$ (expressed with the condition $\sigma_{y}>0$ ). Knowing that $X_{j i}=y$, the multinomial factor is needed to count the remaining $c-1$ pirate symbols in $\boldsymbol{\sigma}$, subtracting 1 from $\sigma_{y}$ (using the $\boldsymbol{e}_{y}$ vector).

$$
\operatorname{Pr}\left[u=g_{1}\left(p_{y}\right) \mid \boldsymbol{p}\right]=\sum_{\boldsymbol{\sigma} \in \mathcal{S}_{q c}} \frac{\sigma_{y}}{c}\left(\begin{array}{c}
c \\
\boldsymbol{\sigma}
\end{array}\right) \boldsymbol{p}^{\boldsymbol{\sigma}} \theta_{y \mid \boldsymbol{\sigma}}
$$


In the last equation we used $\boldsymbol{p}^{\boldsymbol{\sigma}}=p_{y} \boldsymbol{p}^{\boldsymbol{\sigma}-\boldsymbol{e}_{y}}$ and $\left(\begin{array}{c}c-1 \\ \boldsymbol{\sigma}-\boldsymbol{e}_{y}\end{array}\right)=\frac{\sigma_{y}}{c}\left(\begin{array}{c}c \\ \boldsymbol{\sigma}\end{array}\right)$. Then the condition $\sigma_{y}>0$ becomes superfluous and (27) trivially follows. Notice that

$$
p_{y} \frac{\partial T_{y \mid \boldsymbol{p}}}{\partial p_{y}}=p_{y} \frac{\partial}{\partial p_{y}} \sum_{\boldsymbol{\sigma} \in \mathcal{S}_{q c}}\left(\begin{array}{l}
c \\
\boldsymbol{\sigma}
\end{array}\right) \boldsymbol{p}^{\boldsymbol{\sigma}} \theta_{y \mid \boldsymbol{\sigma}}=\sum_{\boldsymbol{\sigma} \in \mathcal{S}_{q c}}\left(\begin{array}{l}
c \\
\boldsymbol{\sigma}
\end{array}\right) \theta_{y \mid \boldsymbol{\sigma}} p_{y} \frac{\partial \boldsymbol{p}^{\boldsymbol{\sigma}}}{\partial p_{y}}=\sum_{\boldsymbol{\sigma} \in \mathcal{S}_{q c}}\left(\begin{array}{l}
c \\
\boldsymbol{\sigma}
\end{array}\right) \theta_{y \mid \boldsymbol{\sigma}} \sigma_{y} \boldsymbol{p}^{\boldsymbol{\sigma}}
$$

proving that $(28)=(27)$ and $(26)=(25)$. Finally, from (64) combined with (63) we have

$$
\psi_{-}(u \mid \boldsymbol{p})=\sum_{y \in \mathcal{Q}} \delta\left(u-g_{0}\left(p_{y}\right)\right)\left(\tau_{y \mid \boldsymbol{p}}-\operatorname{Pr}[X=y \wedge Y=y \mid \boldsymbol{p}]\right) .
$$

This, together with (66), completes the proof.

\section{Proof of Theorem 2}

The full $\psi(u)$, without conditioning, is obtained by taking the expectation over $\boldsymbol{p}$ of $(25)+(27)$.

$$
\psi(u)=\mathbb{E}_{\boldsymbol{p}}[\psi(u \mid \boldsymbol{p})]=\Theta(-u) \mathbb{E}_{\boldsymbol{p}}\left[\psi_{-}(u \mid \boldsymbol{p})\right]+\Theta(u) \mathbb{E}_{\boldsymbol{p}}\left[\psi_{+}(u \mid \boldsymbol{p})\right] .
$$

We first prove (29) starting from $\mathbb{E}_{\boldsymbol{p}}\left[\psi_{-}(u \mid \boldsymbol{p})\right]$ with $\psi_{-}(u \mid \boldsymbol{p})$ as given in (25).

$$
\begin{aligned}
\mathbb{E}_{\boldsymbol{p}}\left[\psi_{-}(u \mid \boldsymbol{p})\right] & =\mathbb{E}_{\boldsymbol{p}}\left[\sum_{y \in \mathcal{Q}} \delta\left(u-g_{0}\left(p_{y}\right)\right) \sum_{\boldsymbol{\sigma} \in \mathcal{S}_{q c}}\left(\begin{array}{l}
c \\
\boldsymbol{\sigma}
\end{array}\right)\left(1-\frac{\sigma_{y}}{c}\right) \boldsymbol{p}^{\boldsymbol{\sigma}} \theta_{y \mid \boldsymbol{\sigma}}\right] \\
& =\sum_{y \in \mathcal{Q}} \sum_{\boldsymbol{\sigma} \in \mathcal{S}_{q c}}\left(\begin{array}{l}
c \\
\boldsymbol{\sigma}
\end{array}\right)\left(1-\frac{\sigma_{y}}{c}\right) \theta_{y \mid \boldsymbol{\sigma}} \mathbb{E}_{\boldsymbol{p}}\left[\delta\left(u-g_{0}\left(p_{y}\right)\right) \boldsymbol{p}^{\boldsymbol{\sigma}}\right] .
\end{aligned}
$$

From Lemma 1 and $\boldsymbol{p}_{\backslash y}^{\boldsymbol{\sigma}_{\backslash y}}=\left(1-p_{y}\right)^{c-\sigma_{y}} \prod_{\alpha \in \mathcal{Q} \backslash\{y\}} t_{\alpha}^{\sigma_{\alpha}}$ we have that

$$
\begin{aligned}
\mathbb{E}_{\boldsymbol{p}}\left[\delta\left(u-g_{0}\left(p_{y}\right)\right) \boldsymbol{p}^{\boldsymbol{\sigma}}\right]= & \frac{1}{B\left(\kappa \mathbf{1}_{q}\right)} \int_{0}^{1} \mathrm{~d} p_{y} \delta\left(u-g_{0}\left(p_{y}\right)\right) p_{y}^{\sigma_{y}+\kappa-1}\left(1-p_{y}\right)^{c-\sigma_{y}+\kappa[q-1]-1} \\
& \int_{0}^{1} \mathrm{~d}^{q-1} \boldsymbol{t} \delta\left(1-\sum_{\beta \in \mathcal{Q} \backslash\{y\}} t_{\beta}\right) \prod_{\alpha \in \mathcal{Q} \backslash\{y\}} t_{\alpha}^{\sigma_{\alpha}+\kappa-1} .
\end{aligned}
$$

The second integral in (72) evaluates to $B\left(\boldsymbol{\sigma}_{\backslash y}+\kappa \mathbf{1}_{q-1}\right)$, having the structure shown in Def. 1. In order to evaluate the $p_{y}$-integral we have to rewrite the delta function into the form $\delta\left(p_{y}-\cdots\right)$. We use the rule

$$
\delta(u-w(p))=\frac{\delta\left(p-w^{\mathrm{inv}}(u)\right)}{|\mathrm{d} w / \mathrm{d} p|}
$$

for any monotonic function $w(p)$. This gives

$$
\delta\left(u-g_{0}(p)\right)=\Theta(-u) \frac{2|u|}{\left(1+u^{2}\right)^{2}} \delta\left(p-\frac{u^{2}}{1+u^{2}}\right) .
$$

We substitute (74) into (72) and solve the integral

$$
\begin{aligned}
\mathbb{E}_{\boldsymbol{p}}\left[\delta\left(u-g_{0}\left(p_{y}\right)\right) \boldsymbol{p}^{\boldsymbol{\sigma}}\right]= & 2|u| \Theta(-u)\left(\frac{1}{1+u^{2}}\right)^{2} \frac{B\left(\boldsymbol{\sigma}_{\backslash y}+\kappa \mathbf{1}_{q-1}\right)}{B\left(\kappa \mathbf{1}_{q}\right)} \int_{0}^{1} \mathrm{~d} p_{y} \delta\left(p_{y}-\frac{u^{2}}{1+u^{2}}\right) \\
& p_{y}^{\sigma_{y}+\kappa-1}\left(1-p_{y}\right)^{c-\sigma_{y}+\kappa[q-1]-1} \\
& =2|u| \Theta(-u)\left(\frac{1}{1+u^{2}}\right)^{2} \frac{B\left(\boldsymbol{\sigma}_{\backslash y}+\kappa \mathbf{1}_{q-1}\right)}{B\left(\kappa \mathbf{1}_{q}\right)}\left(\frac{u^{2}}{1+u^{2}}\right)^{\sigma_{y}+\kappa-1}\left(\frac{1}{1+u^{2}}\right)^{c-\sigma_{y}+\kappa[q-1]-1} \\
& =2 \Theta(-u) \frac{B\left(\boldsymbol{\sigma}_{\backslash y}+\kappa \mathbf{1}_{q-1}\right)}{B\left(\kappa \mathbf{1}_{q}\right)} \frac{\left(u^{2}\right)^{\sigma_{y}+\kappa-1 / 2}}{\left(1+u^{2}\right)^{c+\kappa q}} .
\end{aligned}
$$

Substituting (75) into (71) we have

$$
\mathbb{E}_{\boldsymbol{p}}\left[\psi_{-}(u \mid \boldsymbol{p})\right]=2 \sum_{y \in \mathcal{Q}} \sum_{\boldsymbol{\sigma} \in \mathcal{S}_{q c}}\left(\begin{array}{l}
c \\
\boldsymbol{\sigma}
\end{array}\right)\left(1-\frac{\sigma_{y}}{c}\right) \frac{B\left(\boldsymbol{\sigma}_{\backslash y}+\kappa \mathbf{1}_{q-1}\right)}{B\left(\kappa \mathbf{1}_{q}\right)} \frac{\left(u^{2}\right)^{\sigma_{y}+\kappa-1 / 2}}{\left(1+u^{2}\right)^{c+\kappa q}} \theta_{y \mid \boldsymbol{\sigma}} .
$$


Now we change the summations as follows: the $\sum_{\boldsymbol{\sigma}}$ can be written as $\sum_{b} \sum_{\boldsymbol{x}}$ with $b=\sigma_{y}$ and $\boldsymbol{x}=\boldsymbol{\sigma}_{\backslash y}$, so $\theta_{y \mid \boldsymbol{\sigma}}=\Psi_{b}(\boldsymbol{x})$. Then the summand is a function of only $b$ and $\boldsymbol{x}$, which allows us to write

$$
\sum_{y} \sum_{\sigma}\left(\begin{array}{l}
c \\
\sigma
\end{array}\right) \rightarrow q \sum_{b=0}^{c} \sum_{\boldsymbol{x}}\left(\begin{array}{l}
c \\
b
\end{array}\right)\left(\begin{array}{c}
c-b \\
\boldsymbol{x}
\end{array}\right)
$$

Now we have

$$
\mathbb{E}_{\boldsymbol{p}}\left[\psi_{-}(u \mid \boldsymbol{p})\right]=2 q \sum_{b=0}^{c} \sum_{\boldsymbol{x}}\left(\begin{array}{l}
c \\
b
\end{array}\right)\left(\begin{array}{c}
c-b \\
\boldsymbol{x}
\end{array}\right) \frac{c-b}{c} \frac{B\left(\boldsymbol{x}+\kappa \mathbf{1}_{q-1}\right)}{B\left(\kappa \mathbf{1}_{q}\right)} \frac{\left(u^{2}\right)^{b+\kappa-1 / 2}}{\left(1+u^{2}\right)^{c+\kappa q}} \Psi_{b}(\boldsymbol{x})
$$

where

$$
\begin{aligned}
\sum_{\boldsymbol{x}}\left(\begin{array}{l}
c \\
b
\end{array}\right)\left(\begin{array}{c}
c-b \\
\boldsymbol{x}
\end{array}\right) \frac{B\left(\boldsymbol{x}+\kappa \mathbf{1}_{q-1}\right)}{B\left(\kappa \mathbf{1}_{q}\right)} \Psi_{b}(\boldsymbol{x}) & =\left(\begin{array}{l}
c \\
b
\end{array}\right) \sum_{\boldsymbol{x}}\left(\begin{array}{c}
c-b \\
\boldsymbol{x}
\end{array}\right) \frac{B\left(\boldsymbol{x}+\kappa \mathbf{1}_{q-1}\right)}{B\left(\kappa \mathbf{1}_{q-1}\right) B(\kappa, \kappa[q-1])} \Psi_{b}(\boldsymbol{x}) \\
& =\left(\begin{array}{l}
c \\
b
\end{array}\right) \frac{1}{B(\kappa, \kappa[q-1])} \sum_{\boldsymbol{x}} \mathbb{P}_{q-1}(\boldsymbol{x} \mid b) \Psi_{b}(\boldsymbol{x})=\left(\begin{array}{l}
c \\
b
\end{array}\right) \frac{K_{b}}{B(\kappa, \kappa[q-1])}
\end{aligned}
$$

In the last line we used Definition 3. Substituting (80) into (78) and removing 0 and $c$ from the $b$-range, we have (29). We can use exactly the same steps to obtain (30) from (27). The only significant difference is the delta function which in this case will be

$$
\delta\left(u-g_{1}(p)\right)=\Theta(u) \frac{2 u}{\left(1+u^{2}\right)^{2}} \delta\left(p-\frac{1}{1+u^{2}}\right) .
$$

\section{Proof of consistency check 1}

Integration of (29) and (30) gives

$$
\int_{-\infty}^{\infty} \mathrm{d} u \psi(u)=\frac{2 q}{B(\kappa, \kappa[q-1])} \sum_{b=1}^{c}\left(\begin{array}{l}
c \\
b
\end{array}\right) K_{b}\left[\left(1-\frac{b}{c}\right) \int_{-\infty}^{0} \mathrm{~d} u \frac{\left(u^{2}\right)^{b+\kappa-\frac{1}{2}}}{\left(1+u^{2}\right)^{c+\kappa q}}+\frac{b}{c} \int_{0}^{\infty} \mathrm{d} u \frac{\left(u^{2}\right)^{c-b+\kappa[q-1]-\frac{1}{2}}}{\left(1+u^{2}\right)^{c+\kappa q}}\right] .
$$

Let be $\lambda:=b+\kappa$ and $w:=c-b+\kappa[q-1]$. Applying Lemma 2 we have

$$
\frac{2 q}{B(\kappa, \kappa[q-1])} \sum_{b=1}^{c}\left(\begin{array}{l}
c \\
b
\end{array}\right) K_{b}\left[\left(1-\frac{b}{c}\right) \frac{1}{2} B(\lambda, w)+\frac{b}{c} \frac{1}{2} B(w, \lambda)\right]=\frac{q}{B(\kappa, \kappa[q-1])} \sum_{b=1}^{c}\left(\begin{array}{l}
c \\
b
\end{array}\right) K_{b} B(\lambda, w) .
$$

The result follows applying Lemma 3 followed by Lemma 4.

\section{E Proof of consistency check 2}

Taking (29) and (30), the integral $\int_{-\infty}^{\infty} \mathrm{d} u u \psi(u)$ can be written as

$$
\frac{2 q}{B(\kappa, \kappa[q-1])} \sum_{b=1}^{c}\left(\begin{array}{l}
c \\
b
\end{array}\right) K_{b}\left[\left(1-\frac{b}{c}\right) \int_{-\infty}^{0} \mathrm{~d} u \frac{u\left(u^{2}\right)^{b+\kappa-\frac{1}{2}}}{\left(1+u^{2}\right)^{c+\kappa q}}+\frac{b}{c} \int_{0}^{\infty} \mathrm{d} u \frac{u\left(u^{2}\right)^{c-b+\kappa[q-1]-\frac{1}{2}}}{\left(1+u^{2}\right)^{c+\kappa q}}\right] .
$$

Let $\lambda:=b+\kappa-\frac{1}{2}$ and $w:=c-b+\kappa[q-1]-\frac{1}{2}$. Applying Lemma 2 and the property $\Gamma(x+1)=x \Gamma(x)$ we have

$$
\int_{-\infty}^{\infty} \mathrm{d} u u \psi(u)=\frac{2 q}{B(\kappa, \kappa[q-1])} \sum_{b=1}^{c}\left(\begin{array}{l}
c \\
b
\end{array}\right) K_{b}\left[\left(\frac{b}{c}-1\right) \frac{\Gamma(\lambda) \Gamma(w) \lambda}{2 \Gamma(c+\kappa q)}+\frac{b}{c} \frac{\Gamma(\lambda) \Gamma(w) w}{2 \Gamma(c+\kappa q)}\right] .
$$

To obtain $\tilde{\mu}$ as in $(24)$ we use Lemma 3 to substitute $\left(\begin{array}{l}c \\ b\end{array}\right) \frac{1}{B(\kappa, \kappa[q-1])}$ with $\frac{\mathbb{P}_{1}(b)}{B(\lambda+1 / 2, w+1 / 2)}$. After some simplifications, the result follows. 


\section{F Proof of Lemma 6}

The integral $\int_{-\infty}^{\infty} \mathrm{d} u u^{2} \psi(u)$ can be written as

$$
\frac{2 q}{B(\kappa, \kappa[q-1])} \sum_{b=1}^{c}\left(\begin{array}{l}
c \\
b
\end{array}\right) K_{b}\left[\left(1-\frac{b}{c}\right) \int_{-\infty}^{0} \mathrm{~d} u \frac{u^{2}\left(u^{2}\right)^{b+\kappa-\frac{1}{2}}}{\left(1+u^{2}\right)^{c+\kappa q}}+\frac{b}{c} \int_{0}^{\infty} \mathrm{d} u \frac{u^{2}\left(u^{2}\right)^{c-b+\kappa[q-1]-\frac{1}{2}}}{\left(1+u^{2}\right)^{c+\kappa q}}\right] .
$$

Let $\lambda:=c-b+\kappa[q-1]$ and $w:=b+\kappa$. Applying Lemma 2 with $(2)$ and the property $\Gamma(x+1)=x \Gamma(x)$, we get

$$
\frac{2 q}{B(\kappa, \kappa[q-1])} \sum_{b=1}^{c}\left(\begin{array}{l}
c \\
b
\end{array}\right) K_{b}\left[\left(1-\frac{b}{c}\right) \frac{\Gamma(\lambda-1) \Gamma(w-1) w(w-1)}{2 \Gamma(c+\kappa q)}+\frac{b}{c} \frac{\Gamma(\lambda-1) \Gamma(w-1) \lambda(\lambda-1)}{2 \Gamma(c+\kappa q)}\right] .
$$

Then using (18) we have

$$
\int_{-\infty}^{\infty} \mathrm{d} u u^{2} \psi(u)=q \sum_{b=1}^{c} K_{b} \mathbb{P}_{1}(b)\left[\left(1-\frac{b}{c}\right) \frac{w}{\lambda-1}+\frac{b}{c} \frac{\lambda}{w-1}\right]
$$

and (33) follows after some rewriting.

\section{G Proof of Theorem 5}

We start from Corollary 3 and write a general power series expansion,

$$
\tilde{\chi}(k)=1-(V / 2) k^{2}+\sum_{t=0}^{\infty} \gamma_{t}|k|^{r_{t}},
$$

where the $r_{t} \geq 2+2 \kappa$ are powers and the $\gamma_{t} \in \mathbb{C}$ are coefficients of the form $i^{\beta_{t} \operatorname{sgn} k}$ times a real factor. In this expression the desired relation $\tilde{\chi}(-k)=[\tilde{\chi}(k)]^{*}$ evidently holds, and the properties $\tilde{\chi}(0)=1, \tilde{\chi}^{\prime}(0)=0, \tilde{\chi}^{\prime \prime}(0)=-V$ are clearly present. Then we write

$$
[\tilde{\chi}(k / \sqrt{m})]^{m}=\exp [m \ln \tilde{\chi}(k / \sqrt{m})]=e^{-\frac{V}{2} k^{2}} \exp \left[m \sum_{t=0}^{\infty}\left(\frac{|k|}{\sqrt{m}}\right)^{r_{t}^{\prime}} \delta_{t}\right],
$$

where the powers $r_{t}^{\prime} \geq 2+2 \kappa$ and coefficients $\delta_{t} \propto i^{\beta_{t}^{\prime} \operatorname{sgn} k}$ are obtained (laboriously) by substituting (89) into the Taylor series for the logarithm, $\ln (1+\varepsilon)=\varepsilon-\varepsilon^{2} / 2+\varepsilon^{3} / 3-\varepsilon^{4} / 4+\cdots$. It is worth noting that $m$ disappears from the $k^{2}$ term, but not from the others. Eq. (56) is obtained from (90) by using the Taylor series for the exp function,

$$
\exp \varepsilon=1+\varepsilon+\varepsilon^{2} / 2 !+\varepsilon^{3} / 3 !+\cdots
$$

and (again laboriously) collecting terms with equal powers of $k$. Since we started out with powers $r_{t} \geq 2+2 \kappa$, we end up with powers $\nu_{t} \geq 2+2 \kappa$. Finally, (57) follows by applying Lemma 10 and Lemma 11 to evaluate the integrals that arise when (56) is substituted into Theorem 4. 